\title{
Modelo para Dispersão de Poluentes na Atmosfera com Reflexão nos Contornos Estocásticos
}

\author{
On a Model for Pollutant Dispersion in the Atmosphere with \\ Reflection at the Stochastic Boundaries
}

\author{
Jaqueline Fischer Loeck, Bardo Ernst Josef Bodmann, Marco Túllio Menna Barreto de Vilhena \\ Universidade Federal do Rio Grande do Sul - Programa de Pós-Graduação em Engenharia Mecânica \\ emaildajaque@gmail.com; bardo.bodmann@ufrgs.br; mtmbvilhena@gmail.com
}

\begin{abstract}
Resumo
O presente trabalho apresenta uma análise da influência de componentes estocásticas quando introduzidas em um modelo determinístico. Para tal, a equação de advecção-difusão é resolvida através da transformada de Fourier, cujo dominio é infinito, e em seguida limita-se o intervalo infinito através de reflexões no solo e na camada limite atmosférica. Estas reflexões podem estar refletindo o material poluente de forma completa ou parcial. Os resultados obtidos com este modelo foram comparados com os dados do experimento de Copenhagen.
\end{abstract}

Palavras-chave: Equação de advecção-difusão, reflexão nos contornos, reflexão parcial.

\begin{abstract}
The present work presents an analysis of the influence of stochastic components when introduced in a deterministic model. For this purpose, the advection-diffusion equation is solved via Fourier transform, which has infinite domain, and then the infinite domain is limited by reflection at the ground and at the atmospheric boundary layer limit. These reflections may be reflecting the pollutant material completely or partially. The results obtained with this model were validated with the Copenhagen experimental data.
\end{abstract}

Keywords: Advection-diffusion equation, boundary reflection, partial reflection. 


\section{Introdução}

A qualidade do ar de uma região é elemento essencial para a qualidade de vida da população e do meio ambiente, e é influenciada diretamente pelos níveis de poluição atmosférica. Os desenvolvimentos industrial e tecnológico geram uma emissão excessiva de poluentes, provocando um decaimento na qualidade do ar.

Por este motivo, grupos de pesquisa têm trabalhado no sentido de desenvolver modelos matemáticos que, cada vez mais, se aproximam do fenômeno de dispersão de poluentes na atmosfera, e também em simulações computacionais capazes de estimar o nível de concentração destes poluentes. A partir destas estimativas, pode-se avaliar o impacto ambiental causado pela emissão e adotar medidas de prevenção, com a finalidade de causar o menor dano possível.

Atualmente, o fenômeno da difusão turbulenta na atmosfera não é formulado unicamente, pois não existe um modelo único que explique todos os fenômenos observados. Para estimar o campo de concentração de poluentes, faz-se uso da equação de advecção-difusão, que é obtida através da parametrização dos fluxos turbulentos na equação da continuidade. A equação de advecção-difusão apresenta o problema de fechamento da turbulência, que pode ser solucionado com a teoria $\mathrm{K}$, baseada na hipótese de transporte por gradiente.

Assim, o principal objetivo deste trabalho é investigar o comportamento da solução da equação de advecção-difusão determinística ao utilizar contornos estocásticos, os quais são definidos por reflexões parciais no solo e na camada limite atmosférica. Estas reflexões nos contornos são utilizadas para considerar que o poluente não está confinado entre o solo $(z=0)$ e a camada limite $\left(z=z_{i}\right)$, e pode ultrapassar estas alturas.

\section{Metodologia}

A equação de advecção-difusão com fechamento Fickiano para turbulência modela o fenômeno de dispersão de poluentes, para coeficientes difusivos $K_{x}, K_{y}$ e $K_{z}$ localmente constantes $\left(\mathrm{m}^{2} / \mathrm{s}\right)$, pode ser escrita como (Arya, 1999), (Stull, 1988)

$$
\frac{\partial \bar{c}}{\partial t}+\bar{u} \frac{\partial \bar{c}}{\partial x}+\bar{v} \frac{\partial \bar{c}}{\partial y}+\bar{w} \frac{\partial \bar{c}}{\partial z}=K_{x} \frac{\partial^{2} \bar{c}}{\partial x^{2}}+K_{y} \frac{\partial^{2} \bar{c}}{\partial y^{2}}+K_{z} \frac{\partial^{2} \bar{c}}{\partial z^{2}}+\bar{S},
$$

onde $\bar{c}$ é a concentração média do poluente, $\bar{u}, \bar{v}$ e $\bar{w}$ são as componentes de vento médio $(\mathrm{m} / \mathrm{s})$ orientadas nas direções $x, y$ e $z$, respectivamente, e $\bar{S}$ é o termo fonte.

O termo fonte pode ser descrito por uma condição inicial instantânea, denotada pelas funções delta de Dirac, obtendo assim o modelo conhecido como puff. Para modelar uma liberação contínua de poluentes, integrase no tempo a solução para o modelo puff, obtendo assim a solução para o modelo conhecido com pluma, que é dada por (Seinfeld e Pandis, 2006)

$$
\begin{array}{r}
\bar{C}(x, y, z, t)=\frac{1}{T} \frac{Q}{\sqrt{64 \pi^{3} K_{x} K_{y} K_{z}}} \int_{0}^{t} \frac{1}{\sqrt{(t-\tau)^{3}}} \\
\exp \left\{-\frac{\left[x-x_{0}-\bar{u}(t-\tau)\right]^{2}}{4 K_{x}(t-\tau)}-\frac{\left[y-y_{0}-\bar{v}(t-\tau)\right]^{2}}{4 K_{y}(t-\tau)}\right. \\
\left.-\frac{\left[z-H_{s}-\bar{w}(t-\tau)\right]^{2}}{4 K_{z}(t-\tau)}\right\} d \tau,
\end{array}
$$

onde $x_{0}$ e $y_{0}$ são as coordenadas da localização da fonte no plano cartesiano $(m), H_{s}$ é a altura da fonte $(m)$ e $T$ é uma constante de tempo (s), utilizada para normalizar a concentração.

Esta solução pode ser obtida pela transformada de Fourier, sendo válida para $z \in(-\infty, \infty)$, porém a dispersão é limitada pelo solo e pela camada limite atmosférica, de modo que o intervalo infinito deve ser mapeado para o intervalo finito $z \in\left[0, z_{i}\right]$, onde $z_{i}$ é a altura do topo da camada limite atmosférica. Para isto, serão consideradas infinitas reflexões tanto no solo quanto na camada limite. Estas reflexões podem ser representadas pelas duas sequências

$$
\left.\begin{array}{l}
H_{s} \rightarrow-H_{s}-2 n z_{i} \\
H_{s} \rightarrow H_{s}+2 n z_{i}
\end{array}\right\} \forall n \in \mathbb{Z} .
$$

As sequências apresentadas acima consideram que o poluente que está dispersando, quando atingir o solo ou o topo da camada limite, será refletido por completo de volta para o domínio, sem considerar uma possível permeabilidade parcial, onde parte do material pode se infiltrar no solo ou ultrapassar a camada limite. Considerando esta permeabilidade, serão introduzidos nas sequências que consideram as reflexões (3) os termos $\omega_{c}$ e $\omega_{s}$ que são, respectivamente, os parâmetros de reflexão na camada limite atmosférica e no solo. Estes termos descrevem a quantidade de material poluente que está sendo refletida. Por simplicidade, inicialmente consideraremos $\omega_{c}=\omega_{s}=\omega$, desta maneira, as sequências que representam as reflexões pelos contornos estocásticos, ou seja, que incluem a permeabilidade parcial, são descritas por

$$
\left.\begin{array}{l}
H_{S} \rightarrow-\omega H_{s}-2 n \omega z_{i} \\
H_{s} \rightarrow \omega H_{s}+2 n \omega z_{i}
\end{array}\right\} \forall n \in \mathbb{Z} .
$$

Na solução apresentada anteriormente (2), onde antes apenas era considerada a altura da fonte $H_{s}$, agora serão consideradas estas duas sequências, de maneira que a solução para emissão contínua e reflexão nos contornos estocásticos é dada por 


$$
\begin{array}{r}
\bar{C}(x, y, z, t)=\frac{1}{T} \frac{Q}{\sqrt{64 \pi^{3} K_{x} K_{y} K_{z}}} \int_{0}^{t}\left\{\frac{1}{\sqrt{(t-\tau)^{3}}}\right. \\
\times \exp \left[-\frac{\left[x-x_{0}-\bar{u}(t-\tau)\right]^{2}}{4 K_{x}(t-\tau)}-\frac{\left[y-y_{0}-\bar{v}(t-\tau)\right]^{2}}{4 K_{y}(t-\tau)}\right] \\
\times \sum_{n=-\infty}^{\infty}\left[\exp \left(-\frac{\left[z-\omega H_{s}-2 n \omega z_{i}-\bar{w}(t-\tau)\right]^{2}}{4 K_{z}(t-\tau)}\right)\right. \\
\left.\left.+\exp \left(-\frac{\left[z+\omega H_{s}+2 n \omega z_{i}-\bar{w}(t-\tau)\right]^{2}}{4 K_{z}(t-\tau)}\right)\right]\right\} d \tau .
\end{array}
$$

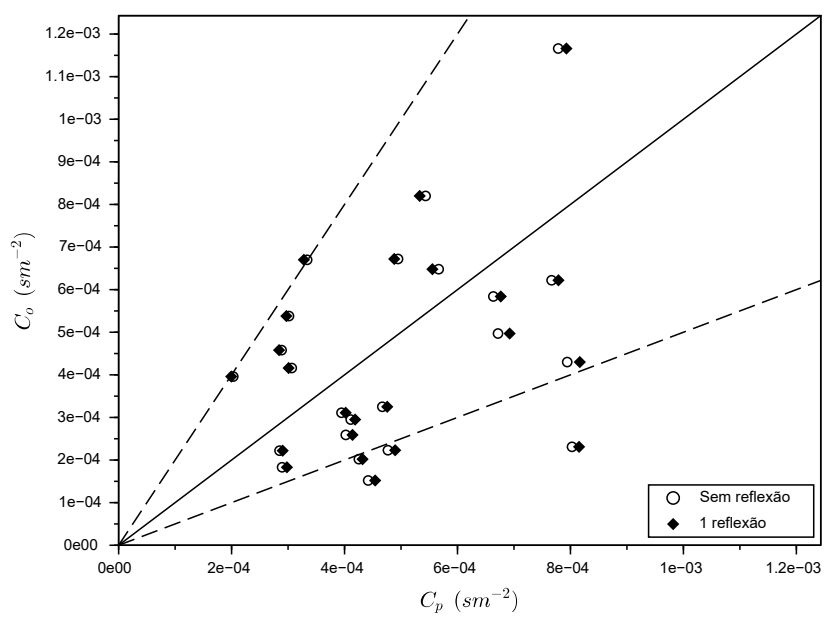

\section{Resultados e Discussões}

Os resultados obtidos foram comparados com os experimentos de Hanford (Doran e Horst, 1985) e de Copenhagen (Gryning e Lyck, 1984) e são preliminares, com o propósito de determinar a contribuição das reflexões na concentração obtida pelo modelo adotado. No presente trabalho, serão apresentados apenas os resultados para o experimento de Copenhagen, uma vez que para o experimento de Hanford foram apresentados no XXXV Congresso Nacional de Matemática Aplicada e Computacional. As principais diferenças entre os dois experimentos são a condição micrometeorológica na qual foram realizados e a altura da fonte, sendo Copenhagen realizado durante condições fortemente convectivas com fonte alta e Hanford durante condições estáveis a quase-neutras com fonte baixa.

Para diferentes valores adotados para $\omega$, foram obtidos números de reflexões distintos, sendo que as reflexões são repetidas até não haver mais contribuição na solução. A Figura 1 apresenta uma comparação entre as concentrações para o modelo sem reflexão e com uma reflexão completa, já as Figuras 2, 3 e 4 apresentam uma comparação do modelo sem reflexão e com 8, 3 e 4 reflexões parciais, respectivamente, porém com parâmetros reflexivos distintos. As Tabelas 1, 2, 3 e 4 apresentam índices estatísticos que indicam a qualidade do modelo (Hanna, 1989), sendo que um bom modelo tem como objetivo NMSE $\rightarrow$ pequeno, $C O R=1-\epsilon, F B \rightarrow|\min |$ e $F S \rightarrow|\min |$, onde ( $\epsilon \rightarrow$ pequeno) representa a diferença entre o modelo determinístico e estocástico. Cabe ressaltar que estes valores nunca são de fato alcançados, pois o modelo utilizado, apesar de utilizar contornos estocásticos, não é capaz de reproduzir exatamente o fenômeno físico em si.
Figura 1: Gráfico de espalhamento das concentrações observadas $C_{o}$ e previstas pelo modelo $C_{p}$ sem reflexão e com 1 reflexão completa $(\omega=1,0)$ para o experimento de Copenhagen.

Tabela 1: Avaliação estatística do modelo utilizando-se o experimento de Copenhagen, $\operatorname{com} \omega=1,0$.

\begin{tabular}{ccccc}
\hline \hline Modelo & NMSE & COR & FB & FS \\
\hline Sem reflexão & 0,26 & 0,38 & $-0,07$ & $-0,28$ \\
Com 1 reflexão & 0,27 & 0,36 & $-0,08$ & 0,25 \\
\hline \hline
\end{tabular}

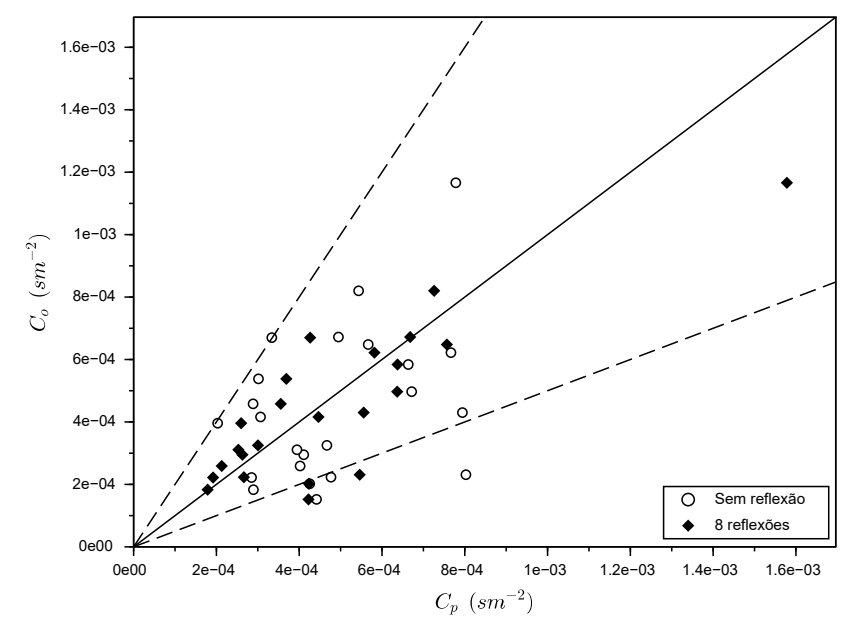

Figura 2: Gráfico de espalhamento das concentrações observadas $C_{0}$ e previstas pelo modelo $C_{p}$ sem reflexão e com 8 reflexões com contornos estocásticos utilizandose o parâmetro $\omega=0,1$ para o experimento de Copenhagen. 
Tabela 2: Avaliação estatística do modelo utilizando-se o experimento de Copenhagen, $\operatorname{com} \omega=0,1$.

\begin{tabular}{ccccc}
\hline \hline Modelo & NMSE & COR & FB & FS \\
\hline Sem reflexão & 0,26 & 0,38 & $-0,07$ & $-0,28$ \\
Com 1 reflexão & 0,2 & 0,67 & 0,16 & 0,4 \\
Com 8 reflexões & 0,11 & 0,81 & $-0,068$ & $-0,19$ \\
\hline \hline
\end{tabular}

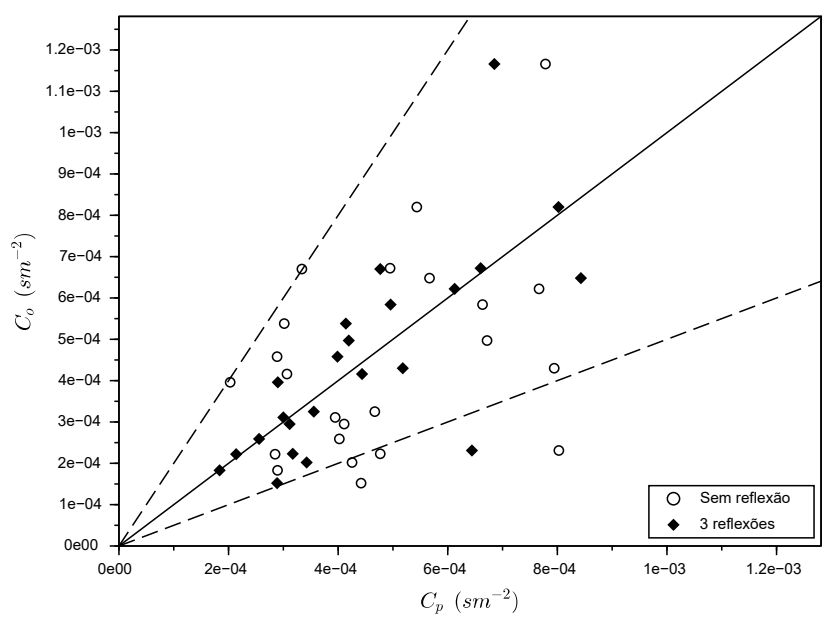

Figura 3: Gráfico de espalhamento das concentrações observadas $C_{o}$ e previstas pelo modelo $C_{p}$ sem reflexão e com 3 reflexões com contornos estocásticos utilizandose o parâmetro $\omega=0,3$ para o experimento de Copenhagen.

Tabela 3: Avaliação estatística do modelo utilizando-se o experimento de Copenhagen, $\operatorname{com} \omega=0,3$.

\begin{tabular}{ccccc}
\hline \hline Modelo & NMSE & COR & FB & FS \\
\hline Sem reflexão & 0,26 & 0,38 & $-0,07$ & $-0,28$ \\
Com 1 reflexão & 0,148 & 0,67 & 0,014 & 0,3 \\
Com 3 reflexões & 0,126 & 0,72 & 0,0047 & 0,28 \\
\hline \hline
\end{tabular}

Tabela 4: Avaliação estatística do modelo utilizando-se o experimento de Copenhagen, $\operatorname{com} \omega=0,2$.

\begin{tabular}{ccccc}
\hline \hline Modelo & NMSE & COR & FB & FS \\
\hline Sem reflexão & 0,26 & 0,38 & $-0,07$ & $-0,28$ \\
Com 1 reflexão & 0,15 & 0,67 & 0,037 & 0,3 \\
Com 4 reflexões & 0,08 & 0,8 & $-0,001$ & 0,17 \\
\hline \hline
\end{tabular}

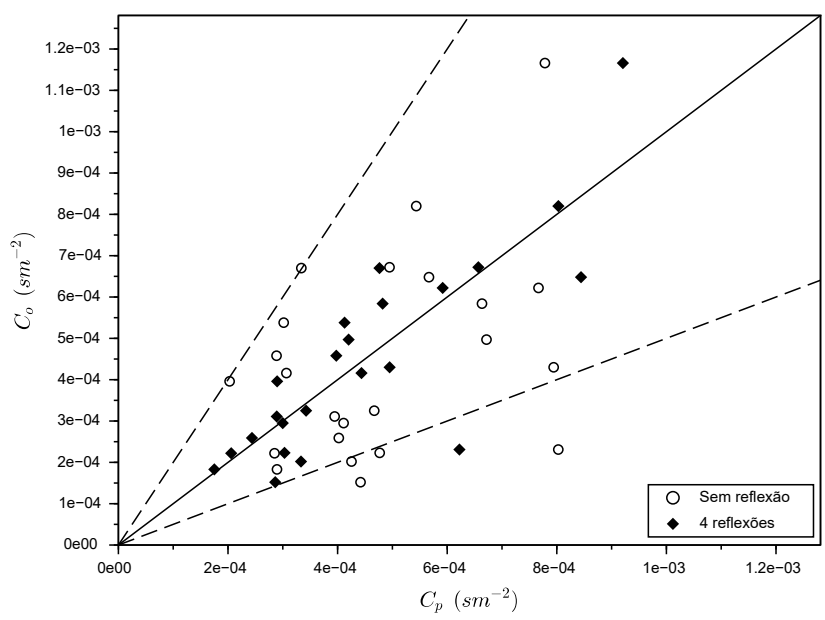

Figura 4: Gráfico de espalhamento das concentrações observadas $C_{o}$ e previstas pelo modelo $C_{p}$ sem reflexão e com 4 reflexões com contornos estocásticos utilizandose o parâmetro $\omega=0,2$ para o experimento de Copenhagen.

\section{Conclusões}

É possível observar que quando são consideradas as reflexões parciais nos contornos estocásticos, os valores obtidos para as concentrações se aproximam consideravelmente dos valores medidos, enquanto na reflexão completa estes valores variam pouco. Ao analisar os índices estatísticos, nota-se que no caso de reflexão completa estes valores se assemelham, já no caso das reflexões parciais obtém-se uma melhora considerável nestes resultados. Desta forma, as reflexões parciais consideram contribuições que o modelo inicial desconsidera.

Assim, o modelo que inclui as reflexões com contornos estocáticos pode ser considerado mais próximo do fenômeno físico analisado em comparação aos modelos que consideram o poluente confinado entre o solo e a camada limite atmosférica, e utilizam como condição de contorno concentração ou fluxo de concentração nulos. Os valores utilizados para o parâmetro de reflexão $\omega$ foram testados e escolhidos conforme obtia-se a melhor solução, por este motivo a próxima etapa para o trabalho será investigar quais valores se adaptam melhor para cada problema.

\section{Agradecimentos}

Agradecemos o suporte financeiro fornecido pelo Conselho Nacional de Desenvolvimento Científico e Tecnológico (CNPq) e pela Coordenação de Aperfeiçoamento de Pessoal de Nível Superior (CAPES). 


\section{Referências}

Arya, S. P. (1999). Air pollution meteorology and dispersion. Oxford University Press, New York.

Doran, J. C., Horst, T. W. (1985). An evaluation of gaussian plume depletion models with dual-tracer field measurements. Atmospheric Environment, 19, 939-951.

Gryning, S. E., Lyck, E. (1984). Atmospheric dispersion from elevated sources in an urban area: Comparison between tracer experiments and model calculations. Journal of Climate and Applied Meteorology, 23(4), 651660.

Hanna, S. R. (1989). Confidence limit for air quality models as estimated by bootstrap and jacknife resampling methods. Atmospheric Environment, 23, 13851395.

Seinfeld, J. H., Pandis, S. N. (2006). Atmospheric chemistry and physics: from air pollution to climate change, $2^{\circ}$ edn. John Wiley \& Sons, New Jersey.

Stull, R. B. (1988). An Introduction to Boundary Layer Meteorology. Kluwer Academic Publishers, Dordrecht, Holanda. 